\title{
A COMPARATIVE STUDY FOR PROPELLER BLADE DESIGN
}

\begin{abstract}
O. de Almeida,
F. C. de Miranda, and $O$. F. Neto

Universidade Federal de Uberlândia Faculdade de Engenharia Mecânica Avenida João Naves de Ávila, 2121 Uberlândia, Minas Gerais, Brasil odenir.almeida@ufu.br

Received: July 07, 2016 Revised: August 10,2016 Accepted: October 28, 2016

ABSTRACT

This work presents a comparative study between two propeller design methods for aeronautical application, with emphasis on its main element, the blade. The first method is an empirical approach based on graphical distribution of design parameters of a propeller and consists on a sequence of steps which starts from defined value for parameters like flight speed, propeller RPM, etc; with a view to obtain others dimensional parameters (diameter, twisting angle, etc) for a propeller to be used on a general aviation aircraft, with the goal to achieve certain performance target. According to the author of this method, the design of a propeller should be seen more as an art rather than exact science. The second method is well known by the aeronautical industry and called "method or theory of blade element". This theory consider a propeller blade as a twisted wing, for which the quantities of interest to be obtained are the aerodynamics reactions, lift and drag, which are a function of the airfoil characteristics (treated as aerodynamic coefficients, $\mathrm{cl}$ for lift and cd for drag) for each section along blades length, twist angle, Mach, etc. For obtaining the propeller value of interest, the number of blades must also be considered. As an application for the study it was used a tri-blade propeller which equips an airplane for general aviation, that can carry 4 occupants flying at 170 Knots. The first aim of this study was to compare the results provided by the empirical method against the BET (Blade Element Theory). A secondary objective was to extend the empirical method in the design of a propeller for use on a closed circuit wind tunnel, once verified the consistency of obtained results as aimed on the first part of this study. Although the results were favorable, showing that both methods provide similar results, the study showed that the empirical method is not valid for operating and constructive conditions set for conditions like the defined for this wind tunnel, once for this type of application, the design parameters extrapolates the minimum and maximum limits established in the empirical method, providing extremely inconsistent results.

Keywords: propeller, blade, wind, tunnel, airplane
\end{abstract}

\section{NOMENCLATURE}

$\begin{array}{ll}\mathrm{p} & \text { pressure, } \mathrm{N} / \mathrm{m}^{2} \\ \mathrm{~T} & \text { aircraft thrust } \\ \mathrm{V} & \text { speed of aircraft in m.p.h } \\ \mathrm{t} & \text { time, } \mathrm{s} \\ \mathrm{N} & \text { R.P.M } \\ \mathrm{N} & \text { revs per second } \\ \mathrm{P} / \mathrm{D} & \text { pitch/diameter ratio } \\ \mathrm{J} & \text { advance ratio } \\ \mathrm{A} & \text { actuator disk area } \\ \mathrm{B} & \text { number of blades } \\ \mathrm{u}_{1}, \mathrm{u}_{2}, \mathrm{u}_{3} & \text { velocity components, } \mathrm{m} / \mathrm{s} \\ \mathrm{X}, \mathrm{y}, \mathrm{Z} & \text { cartesian coordinates, } \mathrm{m}\end{array}$

\section{Greek symbols}

$v$ fluid kinematic viscosity, $\mathrm{m}^{2} / \mathrm{s}$

$\rho \quad$ density, $\mathrm{kg} / \mathrm{m}^{3}$

$\eta \quad$ efficiency

\section{Subscripts}

$\infty$

free stream

\section{INTRODUCTION}

The design of a propeller is a complex issue in the aeronautical field and some people affirm that it's a technical procedure and other affirms that is an art approach. Considering the propeller applications at mobility engineering, it is clear the importance of the design technics for this purpose, especially regarding its accuracy, once this is a non-steady aerodynamic application, what introduce a great challenge on developing a new propeller (blade). Knowing that, there are basically two methods that provide refined enough results, which are, experimental analysis and the more advanced CFD technics - Roskam \& Lan (1997).

The first purpose of this work is to realize a comparative study between some available theoretical and empirical methods used at the development of a propeller. A comparative study will be performed by comparing the results obtained by classical methods and that obtained by the empirical ones, when designing blades for being used at a small experimental airplane of general aviation category. 
A secondary objective is based on the applicability of these methods for designing a propeller for equipping a low speed subsonic wind tunnel that has been developed for the aeronautical engineering school from Federal University of Uberlândia. This tunnel has as main characteristics closed circuit, test chamber dimensions $(1.7 \mathrm{~m} \times 1.3 \mathrm{~m} \times 3.0 \mathrm{~m})$, estimated maximum test speed of $90 \mathrm{~m} / \mathrm{s} \sim 100 \mathrm{~m} / \mathrm{s}$ and an estimated turbulence intensity of around $0.5 \%$.

\section{THEORY}

This work addressed two graphical methods (empirical modeling) of propeller design which have been developed for application in the amateur aviation. These first two methods treated here were developed for an experienced designer of experimental airplanes, and called for him "empirical method" - Clutton (1990).

The other modeling employed in this work is a complete mathematical method applied for propeller design which is based on the momentum conservation theory (or actuator disk) and the blade element theory, namely combined theory. The description of these methods is given in the next subsections.

\section{EMPIRICAL METHOD (Amateur Aviation)}

This empirical method is based mainly on two parts namely nomographs - Cluton (1990), and is a combination of graphics with lines marked with scales representing parameters like power, airspeed, engine speed, propeller diameter and reference lines. For design a new blade, lines are drawn (manually) connecting three input parameters: engine power, engine speed and flight speed. As outputs, the method provides the propeller diameter, pitch and efficiency.

The second part is equal for both methods, and is done by tracing two perpendicular line, one horizontal representing de circumference length, the other is vertical beginning at the border of the horizontal and represents the pitch. Drawing lines from selected positions at horizontal straight which define the sections along the blade, and connecting at de tip of vertical straight, the angles measured between these straight and the horizontal are the twist angle at each blade section selected. After completion of these steps, is possible to draw the new blade and consequently the new propeller. Figure 1 (Nomograph $\mathrm{n}^{\mathrm{o}}$ 1, parts 1 and 2) and Fig. 2 (Nomograph $n^{\circ}$ 2) obtained from the work of Clutton (1990) show the "NOMOGRAPH" for both methods, while Fig. 3, obtained from the same work, shows the scheme for finding the twist angle for blade sections.

The main difference between Nomograph $\mathrm{n}^{\mathrm{o}} 1$ and $n^{\circ} 2$ is the "envelope" or limits of the parameters, allowing a more consistent result with reality. As described by Cluton (1990) the BHP/RPM curves are often not available, so it is better in some cases to work in a reverse way by taking a value for the diameter from a known successful application. Figure 3 will give BHP/RPM for some popular small engines. The calculations will then give the pitch, efficiency, etc.
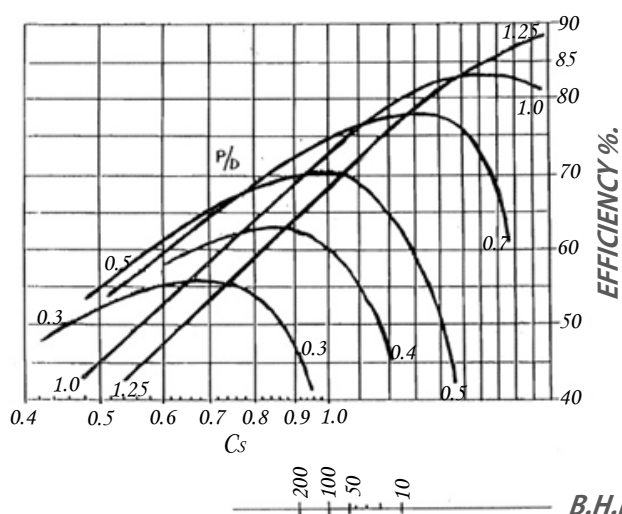
B.H.P.

REF. LINE
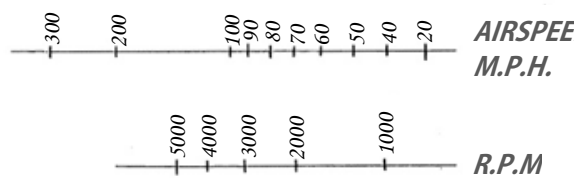

How to use: Connect RPM and Airspeed. Project to Ref. Line. Connect from Ref. Line through BHP to lower edge of Graph. Project Vertically to most favourable P/D curve (see Efficiency).

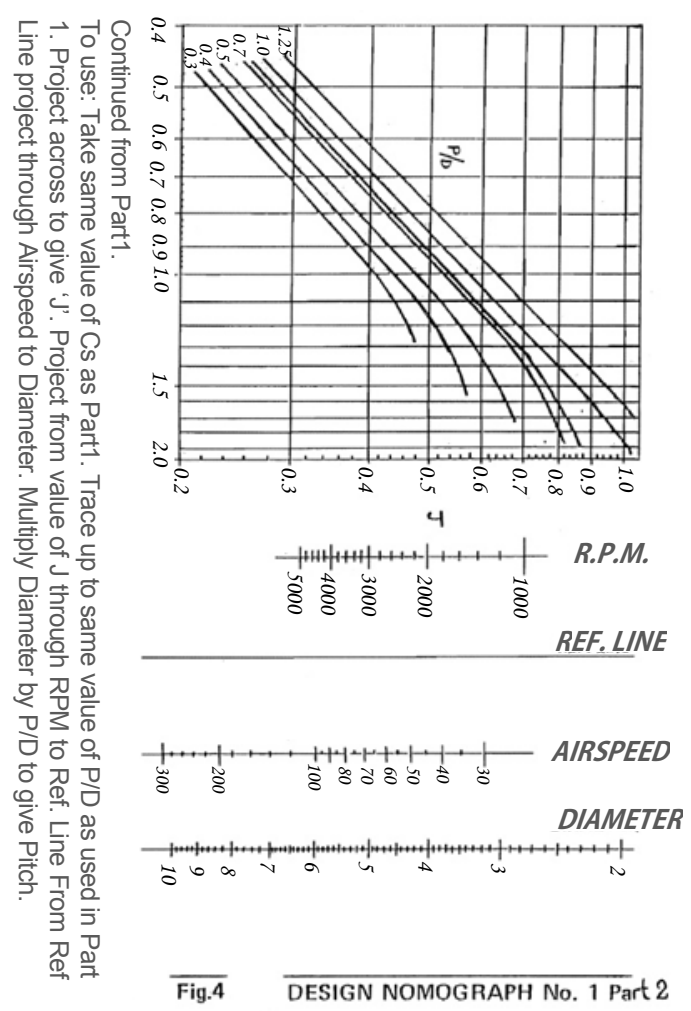

Figure 1. Description of the graphical method called NOMOGRAPH-1 - Clutton (1990). 
Both the nomographs should be used and the slightly differing results tempered with observation and experience - Cluton (1990). Also, Cluton (1990) provided the following useful empirical formula:

$$
D=118 \sqrt[4]{\frac{H P / 100}{\left(\frac{V}{100}\right)\left(\frac{R P M}{1000}\right)^{2}}}
$$

$\mathrm{HP}=$ Horsepower extracted at cruise $\mathrm{V}=$ Cruise speed in $\mathrm{mph}$ $\mathrm{RPM}=\mathrm{RPM}$ at cruise

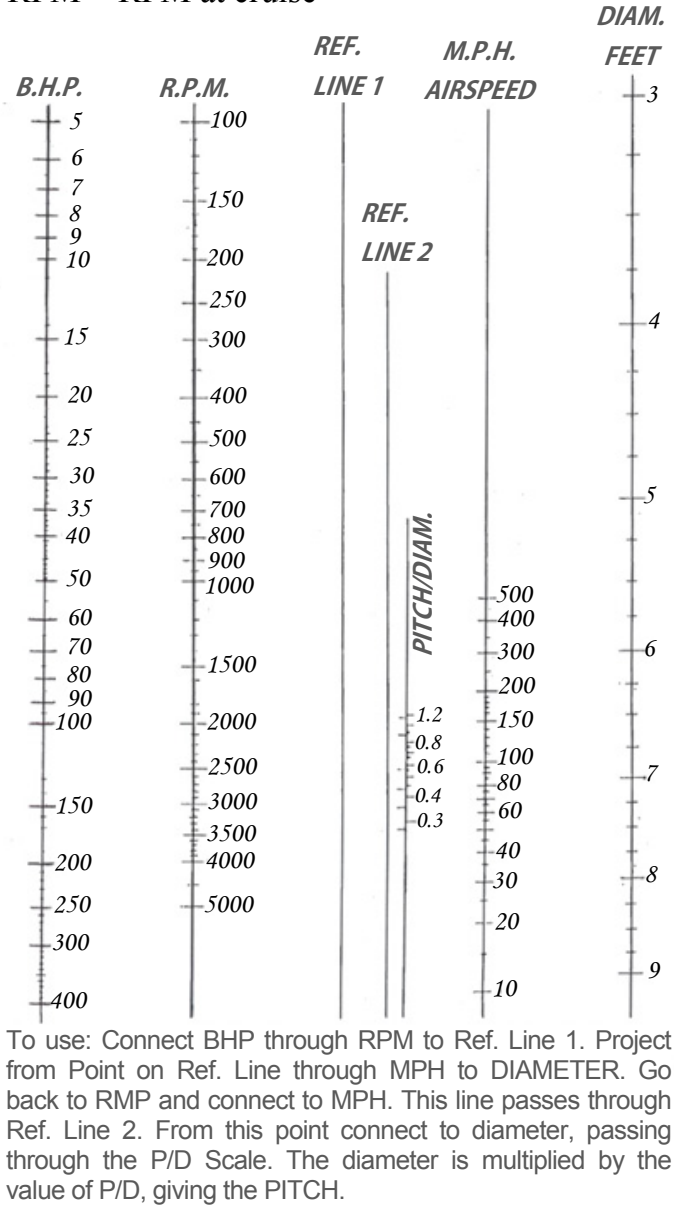

Figure 2. Description of the graphical method called NOMOGRAPH-2 - Clutton (1990).

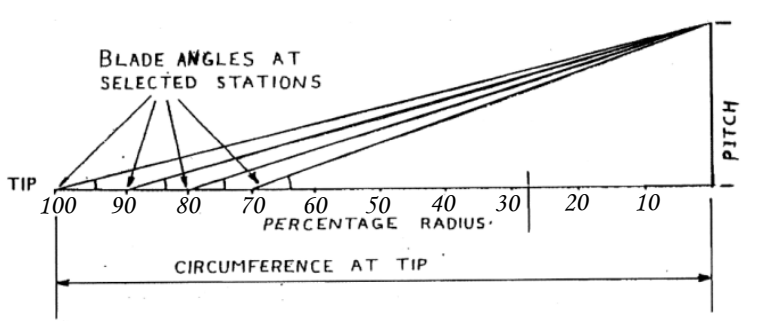

Figure 3. Blade element twist - Clutton (1990).

The author suggests to start the development of a new propeller based on a well-known one, already tested and successfully used on similar application, getting the chord length of the biggest section of the blade, the propeller diameter and blade twist. The shape of the blade is less important and its tip may be square or round, having little impact on its performance.

The author affirm that the profile is not too much important, since some care be taken regarding the leading edge and trailing edge, with the first having a radio not too much sharp and the second not been too thin, mainly for structural reasons. Finally it is important to note that these methods don't allow great accuracy, once it depends on feeling and draw skill of the designer.

\section{MOMENTUM CONSERVATION THEORY}

This theory also called actuator disk theory (Rankine 1865 \& Froude 1885), consider that the propeller provide changes on kinetic energy, static pressure and angular momentum, this results in thrust, absorbed power and maximum efficiency $\eta$.

The momentum conservation method considers the flow as incompressible, uniform and irrotational, where the propeller is substituted by a disk loaded uniformly, infinitely thin and composed by infinite blades.

Consider the speed and pressure before de disk as $V$ and $p$ respectively, and the speed as $V+u$ on the disk, immediately after the disk the pressure is assumed $p+\Delta p$ due the energy increment by the propeller. Applying Bernoulli equation both before the flow and after the disk, is obtained the thrust as shown on equation bellow, where $A$ is the actuator disk area.

$$
T=A \cdot \rho \cdot(V+u) \cdot u^{\prime}
$$

The induced speed $u$ on the wake after the disk due to induced speed $u$ on the disk plane is obtained by the following equation:

$$
2 \cdot u=u^{\prime}
$$

Considering static condition $(\mathrm{V}=0)$ and thrust $\mathrm{T} \neq 0$, is defined the static induced speed:

$$
u_{\text {static }}=u_{s}=\sqrt{\frac{T}{2 A \rho}}
$$

Considering flight condition $(\mathrm{V} \neq 0)$ and thrust $\mathrm{T}=2 \rho \mathrm{A}(\mathrm{V}+\mathrm{u}) \mathrm{u}$, the induced speed is according to:

$$
u=-\frac{V}{2}+\sqrt{\left(\frac{V}{2}\right)^{2}+\frac{T}{2 A \rho}}
$$

For a constant power the ideal efficiency for a free propeller is calculated from the power calculated before and after the propeller, as represented below: 


$$
\eta=\frac{T \cdot(V)}{T \cdot(V+u)}=\frac{V}{V+u}
$$

At real condition of operation the efficiency will never be ideal, once there are losses due swirl wake generated by the propeller, drag generated by de profile of the blade, non-uniform flow, compressibility effect and shadow of the propeller due the fuselage or nacelle. Such losses are not considered on this theory.

\section{BLADE ELEMENT THEORY}

This theory is based on Prandtl lift line theory with some few changes - Pawlowski (1920). On this theory the blade is considered as a too much twisted wing, discretized on many sections, being the profile of each one twisted of an angle $\beta$ and responsible for a parcel of the total aerodynamic loads.

$$
\begin{gathered}
d T=d L \cdot \cos (\varnothing)-d D \cdot \sin (\varnothing) \\
=\frac{\rho \cdot V_{R}^{2} \cdot c \cdot d r \cdot[c l \cdot \cos (\varnothing)-c d \cdot \sin (\varnothing)]}{2} \\
d Q=[d L \cdot \sin \emptyset+d D \cdot \cos (\varnothing)] \\
=0.5 \cdot \rho \cdot V_{R}^{2} \cdot c \cdot r \cdot d r \\
\cdot[c l \cdot \sin (\varnothing)+c d \cdot \cos (\varnothing)] \\
\eta=\frac{V \cdot d T}{\Omega \cdot d Q}=\frac{V \cdot[c l \cdot \cos (\varnothing)-c d \cdot \sin (\varnothing)]}{\Omega \cdot r \cdot[c l \cdot \sin \emptyset+c d \cdot \cos \emptyset]} \\
=\tan (\varnothing) \cdot \frac{[c l \cdot \cos (\varnothing)-c d \cdot \sin (\varnothing)]}{[c l \cdot \sin (\varnothing)+c d \cdot \cos (\varnothing)]}
\end{gathered}
$$

Like on a wing, the aerodynamic load for a given blade element can be decomposed into lift $d L$ and drag $d D$, respectively perpendicular and parallel to resultant speed $V_{R}$, being the thrust $d T$ and the torque $d Q$ for this element obtained from $d L$ and $d D$, as shown in Eq.(6) and Eq.(7) respectively, the aerodynamic efficiency is obtained from $d T$ and $d Q$ as presented on Eq.(8). For these three blade element physical greatness $\Phi$ is the effective attack angle, known as helix angle.

\section{COMBINED THEORY}

The combined theory developed by Weick (1930), may be considered a more complete theory, once it is based on the theories of momentum conservation and blade element. Now, the resultant speed $V_{R}$ presented on blade element theory is corrected including the induced speed $u$ presented on momentum conservation theory and is denoted by $V_{R 0}$, as presented on Eq.(9). This new resultant speed depend on induced angle $\theta$ as depicted on Eq.(10), the terms of position of element along the blade $x$, and total solidity $\sigma_{\text {total }}$ (ratio between the propeller disk area and the wet area of the blades) are presented on equations (11) and (12) respectively.

$$
\mathrm{V}_{\mathrm{R} 0}=\mathrm{V}_{\mathrm{R}} \cdot \cos (\theta)=\left[\frac{2 \cdot \pi \cdot \mathrm{n} \cdot \mathrm{r}}{\cos (\varnothing)}\right] \cdot \cos (\theta)
$$

$$
\begin{gathered}
\theta \\
=\frac{1}{2 \cdot \cos (\varnothing)} \\
\left\{-\left(\sin (\varnothing)+\frac{\left.a_{0} \cdot \sigma_{\text {total }}\right)}{8 \cdot x}\right)\right. \\
\left.+\sqrt{\left(\sin (\emptyset)+\frac{a_{0} \cdot \sigma_{\text {total }}}{8 \cdot x}\right)^{2}+4 \cdot \cos (\varnothing) \cdot \frac{a_{0} \cdot \sigma_{\text {total }}}{8 \cdot x} \cdot(\beta-\emptyset)}\right\} \\
x=\frac{r}{R} \\
\sigma_{\text {total }}=\frac{B \cdot \int_{0}^{R} c(x) d r}{\pi \cdot R^{2}}
\end{gathered}
$$

With: $a_{0}=$ slope of $c l x \alpha$ curve; $V_{t}=$ speed at blade tip; $B=$ number of blades.

Finally for a propeller with a number $B$ of blades, the thrust and torque parcels are as presented by the set of equations, respectively.

$$
\begin{gathered}
d T=B \cdot\left[d L \cdot \cos \left(\emptyset_{0}\right)-d D \cdot \sin \left(\emptyset_{0}\right)\right] \\
=B \cdot \rho \cdot 2 \cdot\left[\frac{\pi \cdot n \cdot r \cdot \cos (\theta)}{\cos (\varnothing)}\right]^{2} \cdot c \cdot d r \\
\cdot\left[c l \cdot \cos \left(\emptyset_{0}\right)-c d \cdot \sin \left(\emptyset_{0}\right)\right] \\
d Q=B \cdot \rho \cdot 2 \cdot r \cdot\left[\frac{\pi \cdot n \cdot r \cdot \cos (\theta)}{\cos (\emptyset)}\right]^{2} \cdot c \cdot d r \\
\cdot\left[c l \cdot \cos \left(\emptyset_{0}\right)+c d \cdot \cos \left(\emptyset_{0}\right)\right]
\end{gathered}
$$

The results provided by this theory will be described in the next subsection and will be denoted by the acronyms B.E.T (Blade Element Theory), for brevity purposes.

\section{RESULTS AND DISCUSSIONS}

On this work four blades were designed and drawn, being three of them for a general aviation airplane and one blade especially designed to be installed on a medium size low speed wind tunnel. This last design has been one extension of the applicability of the theories investigated into this work. All results are shown in sequence:

\section{Propeller blades for General Aviation}

The first design for a propeller to be installed on the proposed airplane was done based on empirical method 1 (NOM-1), the second design was based on empirical method 2 (NOM-2) and the third design (B.E.T) was based on the combined method, using the freeware JavaProp, which apply this method as described by Hepperle (2014) on its user guide. This software was chosen once the results of some 
simulations on this software were study object of the work developed by Barbosa (2009) and considered adequate for such application.

For the airplane propeller design it was considered as reference, the diameter $(1.68 \mathrm{~m})$ of the 3 blades propeller installed at Bumerangue $E X-27$ (experimental airplane) that is equipped with the commercial blade (MTV-12-D/LD168-101b) Figure 3.

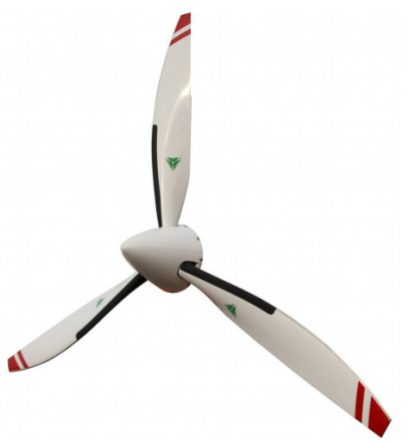

Figure 4. Illustrative example of a MTV propeller blade - (www.mt-propeller.com).

As design parameters for empirical methods were considered the available engine power $(134,226 \mathrm{~W})$, engine speed $(2550 \mathrm{rpm})$ and flight speed $(74.59 \mathrm{~m} / \mathrm{s})$. For the design at JavaProp were considered the preview parameters plus number of blades (3), propeller diameter $(1.798 \mathrm{~m})$, spinner diameter $(0.355 \mathrm{~m})$, air density $\left(\rho=1.008393 \mathrm{~kg} / \mathrm{m}^{3} @\right.$ $1981 \mathrm{~m}$, ISA), kinematic viscosity $\left(v=1.749 \mathrm{e}^{-5} \mathrm{~m}^{2} / \mathrm{s}\right)$ and speed of sound $(\mathrm{a}=332.55 \mathrm{~m} / \mathrm{s})$.

The results are presented in Tab. 1, Tab.2 and Tab.3 for the main design parameter for a blade: relative position along the blade, twist angle and cord length of the element blade.

Table 1. Blade parameters for Blade Element Theory (B.E.T) and nomograph-1 method.

\begin{tabular}{|c|c|c|c|c|c|}
\hline \multirow{2}{*}{$\mathbf{r} / \mathbf{R}(-)$} & \multirow{2}{*}{$\mathbf{r}(\mathbf{m m})$} & \multicolumn{2}{|c|}{$\boldsymbol{\beta}\left(^{(\mathbf{})}\right.$} & \multicolumn{2}{c|}{$\mathbf{c}(\mathbf{m m})$} \\
\cline { 3 - 6 } & & B.E.T & NOM-1 & B.E.T & NOM-1 \\
\hline 0.25 & 224.8 & 60.6 & 57.857 & 68.2 & 137.76 \\
\hline 0.30 & 269.7 & 55.4 & 52.984 & 77.9 & 142.07 \\
\hline 0.40 & 359.6 & 46.6 & 44.847 & 91.6 & 146.86 \\
\hline 0.50 & 449.5 & 39.7 & 38.511 & 96.2 & 146.86 \\
\hline 0.60 & 539.4 & 34.2 & 33.549 & 93.6 & 142.07 \\
\hline 0.70 & 629.3 & 30.0 & 29.614 & 86.3 & 126.48 \\
\hline 0.80 & 719.2 & 27.1 & 26.443 & 71.2 & 106.10 \\
\hline 0.90 & 809.1 & 24.8 & 23.849 & 50.7 & 80.92 \\
\hline 1.00 & 889.0 & 22.9 & 21.696 & 19.1 & 19.10 \\
\hline
\end{tabular}

Table 2. Blade parameters for nomograph-2 method.

\begin{tabular}{|c|c|c|c|}
\hline \multirow{2}{*}{$\mathbf{r} / \mathbf{R}(-)$} & \multirow{2}{*}{$\mathbf{r}(\mathbf{m m})$} & $\left.\boldsymbol{\beta} \mathbf{(}^{\mathbf{9}}\right)$ & $\mathbf{c}(\mathbf{m m})$ \\
\cline { 3 - 4 } & & $\mathbf{N O M - 2}$ & $\mathbf{N O M - 2}$ \\
\hline 0.25 & 249.6 & 49.823 & 152.94 \\
\hline 0.30 & 299.5 & 44.623 & 157.72 \\
\hline 0.40 & 399.3 & 36.509 & 163.04 \\
\hline 0.50 & 499.1 & 60.632 & 163.04 \\
\hline 0.60 & 598.9 & 26.265 & 157.42 \\
\hline 0.70 & 698.8 & 22.927 & 140.42 \\
\hline 0.80 & 798.6 & 20.309 & 117.79 \\
\hline 0.90 & 898.4 & 18.21 & 89.84 \\
\hline
\end{tabular}

\begin{tabular}{|l|l|l|l|}
\hline 1.00 & 998.2 & 16.493 & 19.10 \\
\hline
\end{tabular}

Table 3. Comparative results for blade parameters between blade element theory and nomograph-1 method.

\begin{tabular}{|c|c|c|c|c|c|c|c|}
\hline \multirow[b]{2}{*}{$\mathbf{r} / \mathbf{R}(-)$} & \multirow{2}{*}{$\mathbf{r}(\mathbf{m m})$} & \multicolumn{2}{|c|}{$\beta\left(^{\circ}\right)$} & \multirow{2}{*}{$\begin{array}{c}\text { Error } \\
(\%)\end{array}$} & \multicolumn{2}{|c|}{ c (mm) } & \multirow{2}{*}{$\begin{array}{c}\text { Error } \\
(\%)\end{array}$} \\
\hline & & B.E.T & \begin{tabular}{|l|} 
NOM-1 \\
\end{tabular} & & B.E.T & NOM-1 & \\
\hline 0.25 & 224.8 & 60.6 & 57.857 & 4.53 & 68.2 & 137.76 & -102.00 \\
\hline 0.30 & 269.7 & 55.4 & 52.984 & 4.36 & 77.9 & 142.07 & -82.37 \\
\hline 0.40 & 359.6 & 46.6 & 44.847 & 3.76 & 91.6 & 146.86 & -60.33 \\
\hline 0.50 & 449.5 & 39.7 & 38.511 & 2.99 & 96.2 & 146.86 & -52.66 \\
\hline 0.60 & 539.4 & 34.2 & 33.549 & 1.90 & 93.6 & 142.07 & -51.78 \\
\hline 0.70 & 629.3 & 30.0 & 29.614 & 1.29 & 86.3 & 126.48 & -46.56 \\
\hline 0.80 & 719.2 & 27.1 & 26.443 & 2.42 & 71.2 & 106.10 & -49.02 \\
\hline 0.90 & 809.1 & 24.8 & 23.849 & 3.83 & 50.7 & 80.92 & -59.61 \\
\hline 1.00 & 889.0 & 22.9 & 21.696 & 5.26 & 19.1 & 19.10 & 0.00 \\
\hline
\end{tabular}

As can be noted by the results, the design obtained by the second empirical method can be considered worse than the other two results, because the diameter found $(1.996 \mathrm{~m})$ was far from the reference when compared with the diameter $(1.798 \mathrm{~m})$ obtained by the first empirical method. For the final evaluation, the empirical method 1 was considered. The Tab. 3 presents a comparison between the results of combined method and empirical method 1, as can be observed, the empirical method 1 provide twist angles very similar to that obtained by the combined theory, and even the aerodynamic efficiency is closer, around $83.46 \%$ for combined theory and $87,5 \%$ for empirical method 1 . The results for element chord present a big difference, probably regarding a safety coefficient applied by the developer of the empirical method.

It is important to note that the empirical methods are based at drawing lines over the graphs, using not clear and accurate scale at these graphs, the results obtained by these methods must be considered "estimates" and no refinement process is allowed by the method.

Based at results and discussion presented above, the empirical methods, especially the method 1 may be used for design a propeller intended to be installed on a home build airplane, once precision on results are not required. Figure 5, Fig. 6 and Fig. 7 show the shape obtained for the blades designed by the three methods.

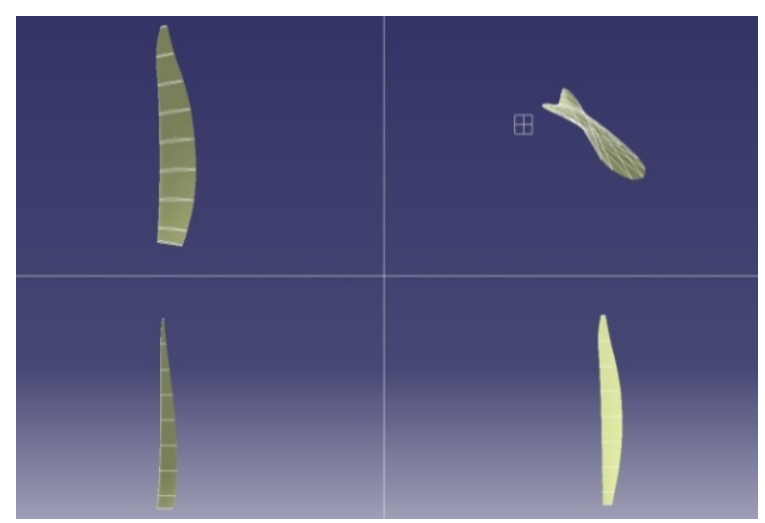

Figure 5. General aviation - Bumerangue blade (B.E.T). 


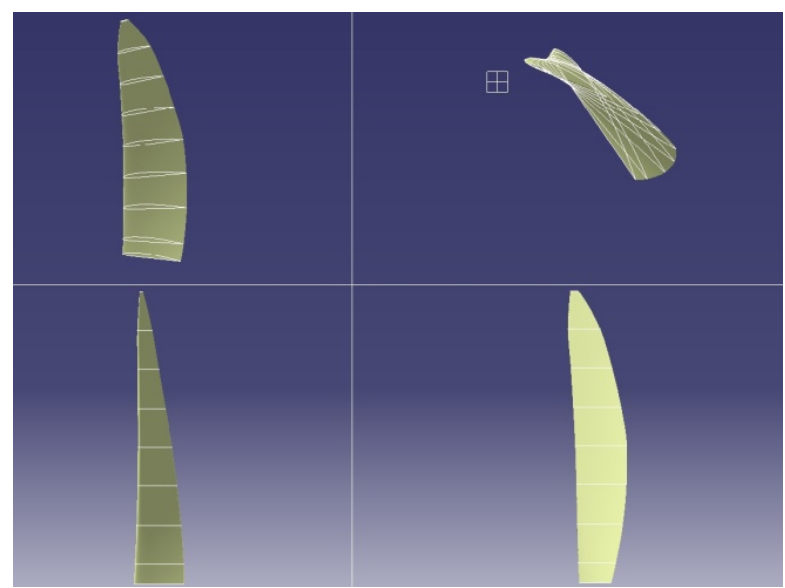

Figure 6. General aviation - Bumerangue blade nomograph-1.

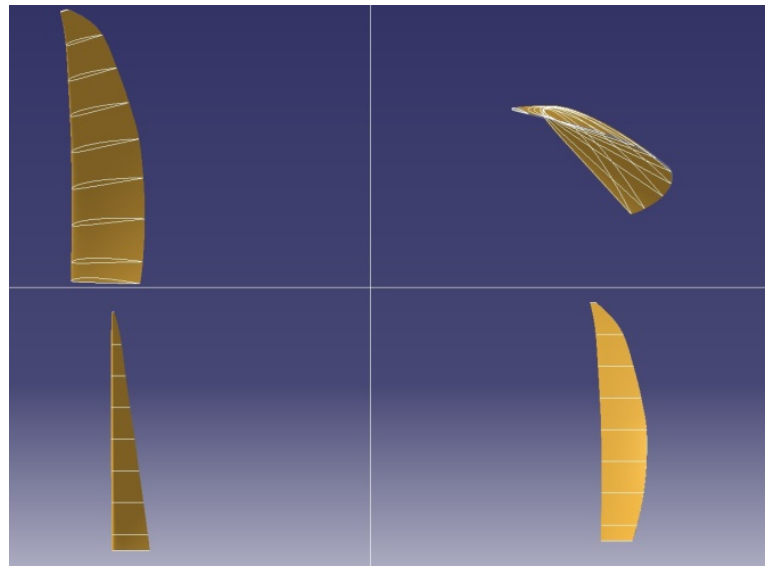

Figure 7. General aviation - Bumerangue blade nomograph-2.

Figure 8 presents side-by-side the shape of the three blades where clearly is seen that the B.E.T results give a slimmer blade than the other.

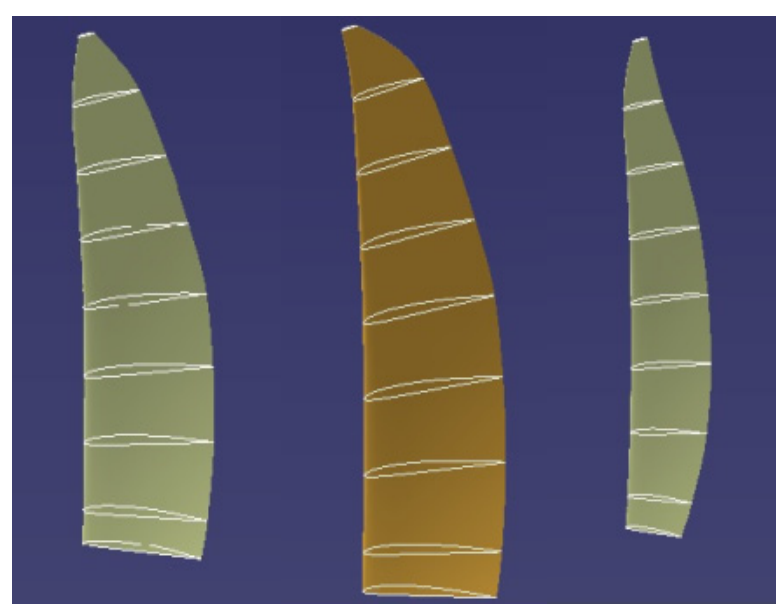

Figure 8. Comparative of blade shape design provided by NOM-1, NOM-2 and B.E.T, respectively.

\section{Wind Tunnel Propeller Blades}

Once the first part of this work presented consistent results, the same steps were followed on the blade design for a medium size wind tunnel. The design parameters are number of blades (8), motor speed $(890 \mathrm{rpm})$, propeller diameter $(2.2 \mathrm{~m})$, spinner diameter $(0.8 \mathrm{~m})$, maximum operational speed at pumping chamber $(48.3 \mathrm{~m} / \mathrm{s})$, maximum available power supplied by the electrical motor $(260 \mathrm{KW})$; and atmospheric parameters: air density $(\rho=$ $\left.1.06428 \mathrm{~kg} / \mathrm{m}^{3} @ 884 \mathrm{~m}, \mathrm{ISA}+\Delta \mathrm{T}=16^{\circ} \mathrm{C}\right)$, kinematic viscosity $\left(v=1.657 \mathrm{e}^{-5} \mathrm{~m}^{2} / \mathrm{s}\right)$ and speed of sound $(\mathrm{a}=$ $346.28 \mathrm{~m} / \mathrm{s})$.

By considering the design parameters, both empirical methods proved to be inadequate for this kind of application, because the lines that must be drawn overtook the limits defined on the graphs, this occurred due to technical characteristics and operational conditions of the wind tunnel, like motor power, motor speed and speed at pumping chamber, which are completely different from that encountered at flight of airplanes. In that sense, the border lines for the empirical methods are applied to amateur aviation where the engine power is restricted not allowing extension of the method for more powerful projects. This is a limitation of the amateur-method (empirical) and no results were obtained.

Therefore the development of the blade propeller for the wind tunnel was based exclusively on the combined method (B.E.T), using the software JavaProp ${ }^{\circledR}$.

Table 4 present the obtained results for the main design parameter for the blade: relative position along the blade, twist angle and cord length of the element blade. Figure 9 shows the views and shape of this blade.

Table 4. Wind tunnel blade parameters for Blade Element Method.

\begin{tabular}{|c|c|c|c|}
\hline \multirow{2}{*}{$\mathbf{r} / \mathbf{R}(\boldsymbol{-})$} & $\mathbf{r}(\mathbf{m m})$ & $\left.\boldsymbol{\beta} \mathbf{(}^{\circ}\right)$ & $\mathbf{c}(\mathbf{m m})$ \\
\cline { 3 - 4 } & & $\mathbf{B . E . T}$ & B.E. $\mathbf{~}$ \\
\hline 0.40 & 440.0 & 63.1 & 190.9 \\
\hline 0.45 & 495.0 & 59.7 & 216.9 \\
\hline 0.50 & 550.0 & 56.5 & 238.2 \\
\hline 0.55 & 605.0 & 53.5 & 258.0 \\
\hline 0.60 & 660.0 & 50.7 & 274.8 \\
\hline 0.65 & 715.0 & 48.1 & 289.0 \\
\hline 0.70 & 770.0 & 45.9 & 297.4 \\
\hline 0.75 & 825.0 & 43.9 & 301.7 \\
\hline 0.80 & 880.0 & 42.1 & 304.2 \\
\hline 0.85 & 935.0 & 40.4 & 305.0 \\
\hline 0.90 & 990.0 & 38.8 & 304.5 \\
\hline 0.95 & 1045.0 & 37.3 & 303.0 \\
\hline 1.00 & 1100.0 & 36.0 & 271.4 \\
\hline
\end{tabular}




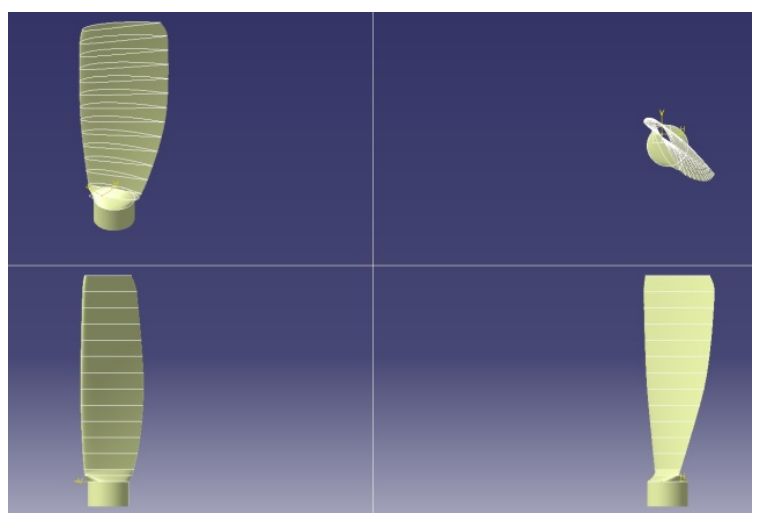

Figure 9. Wind tunnel propeller blade - (B.E.T). Figure 10 illustrates the wind tunnel blade design provided by the application of the B.E.T method.
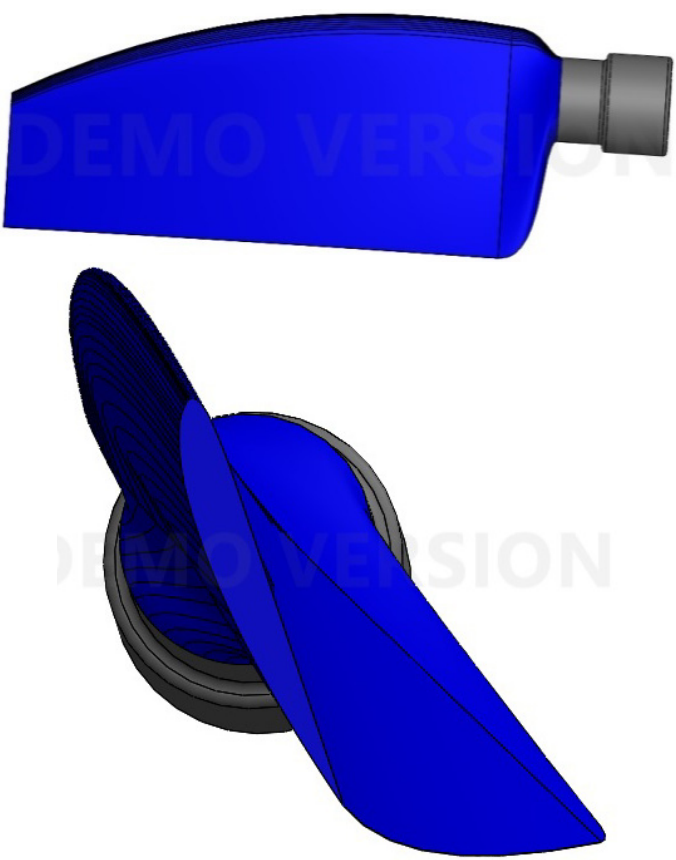

Figure 10. Propeller blade designed by B.E.T for application in a medium-size wind tunnel.

As mentioned at the beginning of this work, the main objective was to validate the application of the empirical methods for design propellers to be applied on two complete different situations which are propelled airplanes (general aviation) and wind tunnel propellers/blades.

By the results obtained, the two empirical methods (NOM-1 and NOM-2) proved to be unappropriated for the second purpose, since the design characteristics are too different from that which those methods were based on.

However, the empirical methods proved to be adequate on designing propeller blades for application on airplanes, especially on home build and sometimes general aviation, once performance accuracy are not required by the market.

On the other hand, the mathematical model described by the combined theory (Blade Element Theory) showed good results for a wide range of application since propeller for low engine power until larger applications with high demanding power output, the case of a medium-size wind tunnel design. This comparative study was also important to illustrate the applicability of such methods at the aeronautical industry, where reasonable estimates could be done with simpler methods providing results without spending cost and time. Nevertheless, where accuracy is needed, designers should go to more refined methods based on the fundamentals aerodynamics (physics).

\section{CONCLUSIONS}

A comparative study between two propeller design methods for aeronautical application has been addressed in this work, with emphasis on its main element, the blade. Two empirical approaches based on graphical distribution of design parameters of a propeller have been evaluated for two distinct aeronautical application: a) propeller design for a general aviation aircraft; $b$ ) extension of the method to design a propeller blade for a medium-size wind tunnel drive-system; These empirical methods have been compared with the combined theory (B.E.T) for propeller design which is a more consistent mathematically and can be used without restrictions for a wide range of applications.

This comparative study showed that reasonable and consistent results can be obtained with both empirical methods and the B.E.T for designing a propeller blade for application in small aircrafts for general aviation. Despite the fact that the empirical methods provided larger shapes for the blades (probably due to safety margins imposed in the design process), it could be applied easily for design without the need of computers and further detailed calculus procedure. In fact, this is one of the great advantages of these empirical methods, which could provide reasonable results at low cost and time solutions.

On the other hand the blade element theory proved itself to be very consistent and reliable for such designing. In fact, the results showed a slimmer blade shape for an applied target efficiency, reducing weight and engine power. Moreover, the methodology could be extended to be applied in the design of blades for using in a medium-size wind tunnel, providing a propeller blade that meets the targets dimensions and efficiency.

\section{ACKNOWLEDGEMENTS}

The authors would like to thank the FINEP $(0138 / 11)$ for funding the wind tunnel design and the administration of Federal University of Uberlândia (UFU) for all support during the phases of this project. Also, the authors recognize the support of 
Fundação de Amparo a Pesquisa do Estado de Minas Gerais - FAPEMIG. Especial thanks are also given to the FABE (Fábrica Brasileira de Aeronaves LTDA).

\section{REFERENCES}

Roskam, J., and Lan, C. E., 1997, Airplane Aerodynamics and Performance, DARcorporation.

Barbosa, F. R., 2009, Design and Analysis on Performance of Optimum Propeller Applying Genetic algorithm, Master Thesis, ITA, São José dos Campos, SP.

Clutton, E., 1990, Propeller Making for Amateur, Tullahoma.

Hepperle, M., 2014, JavaProp, Users Guide.

Pawlowski, F. W.,1920, Théorie Générale de L'Hélice Propulsive, Gauthier-Villars. 